\title{
SEMIGROUPS OF OPERATORS ON LOCALLY CONVEX SPACES
}

\author{
BY \\ V. A. BABALOLA( $\left.{ }^{1}\right)$
}

ABSTRACT. Let $X$ be a complex Hausdorff locally convex topological linear space and $L(X)$ the family of all continuous linear operators on $X$. This paper discusses the generation and perturbation theory for $C_{0}$ semigroups $\{S(\xi): \xi \geqslant 0\} \subset L(X)$ such that for each continuous seminorm $p$ on $X$ there

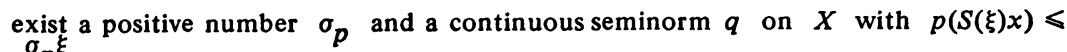
$e^{\sigma_{p} \xi} q(x)$ for all $\xi \geqslant 0$ and $x \in X$.

These semigroups are studied by means of a realization of $X$ as a projective limit of Banach spaces, using certain naturally-defined operators and $C_{0}$ semigroups on these Banach spaces to connect the present results to the classical HilleYosida-Phillips theory.

- Introduction. Strongly continuous one-parameter semigroups of operators (hereafter, $C_{0}$ semigroups) on complex Hausdorff locally convex topological linear spaces (hereafter lcs) have been discussed by many authors under varying restrictions. First, Schwartz [10] imposed the hypothesis of global equicontinuity $(\{S(\xi): \xi \geqslant 0\}$ equicontinuous in $L(X))$ which he employed to recapture the integral representation of the resolvent of the infinitesimal generator, thus reducing the treatment essentially to the classical situation in the Banach space. Other authors (e.g. Miyadera [6], Yosida [13], Komatsu [3], Moore [7], [8]) have also discussed various classes of $C_{0}$ semigroups on lcs retaining one form of global equicontinuity or the other. Waelbroeck [12] studied a class of $C^{\infty}$ semigroups in algebras with complete bounded structure, using a notion of "asymptotic resolvent" and his operational calculus which typically applies to locally equicontinuous

Received by the editors March 14, 1972 and, in revised form, August 29, 1973 and November 29, 1973.

AMS (MOS) subject classifications (1970). Primary 47A55, 47D05; Secondary 46A05.

Key words and phrases. One-parameter $C_{0}$ semigroups, infinitesimal generator, semigroup generation, compartmentalized operators, $L_{A}(X)$-operators, projective limit operators, generator-perturbation by $L_{A}(X)$-operators, Hille-Yosida-Phillips theorem.

(1) Most of the results in this paper form part of the author's Ph.D. thesis at the University of Ibadan, Nigeria; written under the direction of Professor A. Olubummo to whom the author is greatly indebted. I am also very grateful to Professor R. T. Moore for his many useful suggestions. This research was partially supported by an N.S.F. grant.

Copyright $\odot 1974$, American Mathematical Society 
semigroups on an lcs whose infinitesimal generators are continuous. The recent paper of Komura [4], in effect, removes this restriction on the generator, treating general locally equicontinuous semigroups by means of vector-valued distribution theory and "distribution resolvents." Ouchi [9] has just published an article simplifying considerably Komura's work rather in the spirit of Waelbroeck's "asymptotic resolvent" ideas. In these last three papers, the classical resolvent of an operator is not necessarily present, and the replacements for it are of a sufficiently technical nature that their application to the examples that arise in practice seems unnecessarily clumsy and inefficient.

In this paper, we introduce a class of semigroups which nontrivially generalizes the globally equicontinuous classes discussed in the first six references mentioned above, yet is considerably more tractable by classical methods than the locally equicontinuous semigroups considered by Waelbroeck, Komura and Ouchi. Our semigroups, called $\left(C_{0}, 1\right)$ semigroups, can be characterized as $C_{0}$ semigroups $\{S(\xi): \xi \geqslant 0\}$ on $X$ such that for each continuous seminorm $p$ on $X$ there exist a positive number $\sigma_{p}$ and a continuous seminorm $q$ on $X$ satisfying $p(S(\xi) x) \leqslant e^{\sigma} p^{\xi} q(x)$ for all $x \in X$ and $\xi \geqslant 0$ :

As an example, consider the Fréchet space $X=S=S(\mathbf{R})$ of all rapidly decreasing test functions whose topology is determined by the seminorms $\left\{p_{m n}\right\}$ defined as follows. Let

$$
(M f)(x)=x f(x), \quad(D f)(x)=\frac{d}{d x} f(x) \text { and }\|f\|=\left(\int_{\mathbf{R}}|f(x)|^{2} d x\right)^{1 / 2} .
$$

Then $p_{m n}(f)=\left\|M^{m} D^{n} f\right\|$ for all $m, n \geqslant 0$. Consider the group $G=\{S(\xi)$ : $-\infty<\xi<\infty\}$ defined by $(S(\xi) f)(x)=f\left(e^{\xi} x\right)$ for all $f \in S$. Note that $p_{m n}(S(\xi) f)=e^{(n-m-1 / 2) \xi} p_{m n}(f) . G$ is a $\left(C_{0}, 1\right)$ group but for no $\omega<\infty$ is $\left\{e^{-\omega|\xi|} S(\xi):|\xi| \geqslant 0\right\}$ equicontinuous. The improper integral $\int_{0}^{\infty} e^{-\lambda \xi} S(\xi) f d \xi$ is not convergent for any $\lambda$ and the infinitesimal generator $x d / d x$ of $G$ does not have a resolvent in $S$. Note further than the adjoint of a $\left(C_{0}, 1\right)$ group need not be $\left(C_{0}, 1\right)$ even on reflexive spaces. For example, the adjoint $G^{*}$ of $G$ is not of class $\left(C_{0}, 1\right)$. (Details for these claims are supplied in $\S 5$ below.)

Although mathematical tradition permits mathematics for its own sake, without demanding applicability as a necessary index of value, the pertinence of the results presented below to other areas of mathematics forms part of the basis for its interest. Operator semigroup theory on Banach spaces has played an important role in the development of the theory of partial differential equations on Banach spaces. An lcs being a more natural domain for a differential operator, partial differential equations are being currently studied on lcs, e.g. spaces of continuous functions, test functions and distributions with nonnormable lcs topology. It is therefore in order to extend the Banach space theory of operator semigroups to 
the lcs case so as to make it adequately available for application to partial differential equations at this level.

1. Preliminaries. We take $\left\{p_{\alpha}: \alpha \in A\right\}$ as a saturated family of continuous seminorms on $X$ such that the family $\left\{\epsilon V_{\alpha}: \alpha \in A, \epsilon>0\right\}, \quad\left(V_{\alpha}=\{x \in X\right.$ : $\left.\left.p_{\alpha}(x) \leqslant 1\right\}\right), \quad$ is a base of neighborhoods at the origin for the topology of $X$.

1.1 Definition. $L_{\mathrm{A}}(X)$ denotes the family of linear operators $T: X \rightarrow X$ such that there exist positive numbers $\lambda_{\alpha, T}$ with

$$
T V_{\alpha} \subset \lambda_{\alpha, T} V_{\alpha} \text { for all } \alpha \in A \text {. }
$$

Note that every $T \in L_{A}(X)$ is continuous and is such that

$$
p_{\alpha}(T x) \leqslant \lambda_{\alpha, T} p_{\alpha}(x) \text { for all } x \in X \text { and } \alpha \in A \text {. }
$$

Furthermore, it is not difficult to see that the family of linear operators $T: X \rightarrow$ $X$ such that there exist complex or real numbers $\lambda_{\alpha, T}$ with $T V_{\alpha} \subset \lambda_{\alpha, T} V_{\alpha}$ for all $\alpha \in A$ is identical with $L_{A}(X)$, since each $V_{\alpha}$ is balanced. It follows that $L_{A}(X)$ satisfies the definition of the family $L\left(U_{i}, U_{i}\right)$ defined in $[5$, p. 66]. Thus with addition defined pointwise and multiplication by composition, $L_{A}(X)$ becomes an algebra.

Now, for each $p_{\alpha} \in\left\{p_{\alpha}: \alpha \in A\right\}$, the real-valued function $P_{\alpha}$ on $L_{A}(X)$, defined by

$$
\begin{aligned}
P_{\alpha}(T) & =\inf \left\{\lambda_{\alpha, T}: p_{\alpha}(T x) \leqslant \lambda_{\alpha, T} p_{\alpha}(x) \text { for all } x \in X\right\} \\
& =\sup _{x \in X, p_{\alpha}(x) \leqslant 1} p_{\alpha}(T x),
\end{aligned}
$$

is a seminorm with the additional property that

$$
P_{\alpha}\left(T_{1} T_{2}\right) \leqslant P_{\alpha}\left(T_{1}\right) P_{\alpha}\left(T_{2}\right), \quad T_{1}, T_{2} \in L_{A}(X) .
$$

It is also true, from (1.3) and (1.4), that

$$
p_{\alpha}(T x) \leqslant P_{\alpha}(T) p_{\alpha}(x) \text { for all } T \in L_{A}(X), \quad \alpha \in A \text { and } x \in X
$$

Set

$$
\gamma_{\alpha}=\left\{T \in L_{\mathrm{A}}(X): T V_{\alpha} \subset V_{\alpha}\right\} .
$$

As in [5, Definition A. 3, p. 67] we impose on $L_{A}(X)$ the topology having the family

$$
\left\{\epsilon \bigcap_{j=1}^{m} \gamma_{\alpha_{j}}: \epsilon>0, \alpha_{j} \in A, m \text { is finite }\right\}
$$


as base of neighborhoods at the origin. Observe that

$$
\gamma_{\alpha}=\left\{T \in L_{\mathrm{A}}(X): P_{\alpha}(T) \leqslant 1\right\} .
$$

Thus the topology on $L_{A}(X)$ is defined by the family $\left\{P_{\alpha}: \alpha \in A\right\}$ of seminorms on $L_{A}(X)$. Now from [5, Proposition 2.4(j)] under this topology $L_{A}(X)$ becomes a Hausdorff locally multiplicatively-convex topological algebra. We shall understand $L_{\mathrm{A}}(X)$ to mean this topological algebra in the sequel. $L_{\mathrm{A}}(X)$ is complete if $X$ is complete [5, Proposition A. 4].

We next consider other spaces $X_{\alpha}$ and $L\left(X_{\alpha}\right)$.

For each $\alpha$, let $x_{\alpha}$ be the coset of $x$ in the quotient space $X / p_{\alpha}^{-1}(0)$. Put

$$
\left\|x_{\alpha}\right\|=p_{\alpha}(x) \text { for each } x \in X \text {. }
$$

So defined $\|\cdot\|$ is a norm on $X / p_{\alpha}^{-1}(0)$. Under the topology induced by $\|\cdot\|$ the normed linear space formed by the elements of $X / p_{\alpha}^{-1}(0)$ is denoted by $X_{\alpha}$.

For each $\alpha$ the natural homomorphism $\pi_{\alpha}$ of $X$ onto $X_{\alpha}$, defined by $\pi_{\alpha}(x)=x_{\alpha}$ for each $x \in X$, is continuous.

We shall next relate $L_{A}(X)$ to the uniform norm algebra $L\left(X_{\alpha}\right)$. First note that as a consequence of (1.3)

$$
T \in L_{\mathrm{A}}(X) \text { implies } T\left(p_{\alpha}^{-1}(0)\right) \subset p_{\alpha}^{-1}(0) \text { for each } \alpha \in \mathrm{A} \text {. }
$$

1.12 Proposition. Let $\alpha \in A$, and let $S \in L_{\mathrm{A}}(X)$. Then the operator $S_{\alpha}: X_{\alpha} \rightarrow X_{\alpha}$, defined by $S_{\alpha} x_{\alpha}=(S x)_{\alpha}, x_{\alpha} \in X_{\alpha}$, is in $L\left(X_{\alpha}\right)$.

Proof. Suppose $(x-y) \in p_{\alpha}^{-1}(0)$. Then

$$
S_{\alpha}(x-y)_{\alpha}=(S(x-y))_{\alpha}=(0)_{\alpha}
$$

by (1.11). Also

$$
(S(x-y))_{\alpha}=(S x-S y)_{\alpha}=(S x)_{\alpha}-(S y)_{\alpha}=S_{\alpha} x_{\alpha}-S_{\alpha} y_{\alpha} .
$$

Thus $S_{\alpha} x_{\alpha}=S_{\alpha} y_{\alpha}$, that is $S_{\alpha}$ is well defined. Now since $S \in L_{A}(X)$, $p_{\alpha}(S x) \leqslant P_{\alpha}(S) p_{\alpha}(x)$ for all $x \in X$ (see (1.6)). Then from the definition of $S_{\alpha}$ and (1.10) we have

$$
\left\|S_{\alpha} x_{\alpha}\right\|=\left\|(S x)_{\alpha}\right\|=p_{\alpha}(S x) \leqslant P_{\alpha}(S) p_{\alpha}(x)=P_{\alpha}(S)\left\|x_{\alpha}\right\|
$$

for all $x_{\alpha} \in X_{\alpha}$. Therefore $S_{\alpha}$ is bounded on $X_{\alpha}$.

Linearity of $S_{\alpha}$ is a consequence of the linearity of $S$ and the natural homomorphism $\pi_{\alpha}$, and the proof is complete. 
For convenience, call an operator $S: D(S) \subset X \rightarrow X$ which is such that, for each $\alpha$, the operator $S_{\alpha}: \pi_{\alpha}(D(S)) \rightarrow X_{\alpha}$, given by $S_{\alpha} x_{\alpha}=(S x)_{\alpha}, \quad x_{\alpha} \in$ $\pi_{\alpha}(D(S))$, is well defined, a compartmentalized operator (w.r.t. $\left\{p_{\alpha}: \alpha \in A\right\}$ ). Thus $S \in L_{A}(X)$ is a compartmentalized operator. Moreover, for $S \in L_{A}(X)$, $S_{\alpha}$ is uniquely extensible to a bounded linear operator $\bar{S}_{\alpha}$ on the completion $\bar{X}_{\alpha}$ of $X_{\alpha}$ such that

$$
\left\|\bar{S}_{\alpha}\right\|=\left\|S_{\alpha}\right\|=\sup _{\left\|x_{\alpha}\right\| \leqslant 1}\left\|S_{\alpha} x_{\alpha}\right\|=P_{\alpha}(S) .
$$

Finally we identify $L_{\mathrm{A}}(X)$ with projective families of operators.

Now order the index set A by $\alpha \geqslant \alpha^{\prime}$ iff $V_{\alpha} \subset V_{\alpha^{\prime}}$. Define, for $\alpha \geqslant \alpha^{\prime}$, an operator $\pi_{\alpha^{\prime} \alpha}: X_{\alpha} \rightarrow X_{\alpha^{\prime}}$ by $\pi_{\alpha^{\prime} \alpha}\left(x_{\alpha}\right)=x_{\alpha^{\prime}} \cdot \pi_{\alpha^{\prime} \alpha}$ is a continuous homomorphism of $X_{\alpha}$ onto $X_{\alpha^{\prime}}$. Consequently it can be uniquely extended to a continuous homomorphism $\pi_{\alpha^{\prime} \alpha}$ of $\bar{X}_{\alpha}$ into $\bar{X}_{\alpha^{\prime}}$. Like this, $\left\{\bar{X}_{\alpha}: \alpha \in A\right\}$ is a projective family of Banach spaces and its projective limit is $X$.

Suppose, in general, that $\left\{Z_{\alpha}: \alpha \in A\right\}$ is a projective family of Banach spaces with $A$ upward directed by " $\geqslant$ ". Let $\pi_{\alpha \beta}: Z_{\beta} \rightarrow Z_{\alpha} \quad(\beta \geqslant \alpha)$, be the associated continuous maps satisfying $\pi_{\alpha \beta} \pi_{\beta \gamma}=\pi_{\alpha \gamma}(\beta \geqslant \alpha, \gamma \geqslant \beta)$. Denote by $Z$ the projective limit (endowed with the projective topology), where, for each $z \in Z, \pi_{\alpha}(z)=z_{\alpha}$ and $\pi_{\beta \alpha}\left(z_{\alpha}\right)=z_{\beta}(\alpha \geqslant \beta) . Z$ is a complete lcs. Call a projective family of Banach spaces saturated if every finite product of members is still a member. We shall always assume that our projective family of Banach spaces is saturated. There is no restriction on the projective limit in so doing. Otherwise, for each finite subset $A$ of $A$, define $p_{A}$ by

$$
p_{A}(z)=\max _{\alpha \in A}\left\|z_{\alpha}\right\| \text { for all } z \in Z
$$

The $\bar{Z}_{A}$ 's can then be included in the projective family without changing the projective limit, thus obtaining a saturated projective family. Note that $\left\{\bar{X}_{\alpha}\right.$ : $\alpha \in A\}$ above satisfies our requirement of being saturated.

For each $\alpha \in A$, let $S_{\alpha}$ be a linear operator from $D\left(S_{\alpha}\right) \subset Z_{\alpha}$ into $Z_{\alpha}$. Call $\left\{S_{\alpha}: \alpha \in A\right\}$ a (saturated) projective family of operators iff for $z_{\beta} \in D\left(S_{\beta}\right)$ and $\beta \geqslant \alpha, S_{\alpha}\left(\pi_{\alpha \beta} z_{\beta}\right)=\pi_{\alpha \beta}\left(S_{\beta} z_{\beta}\right)$. For such a family define $S$ on the projective limit $D(S)$ of $\left\{D\left(S_{\alpha}\right): \alpha \in A\right\}$ by $\pi_{\alpha}(S z)=S_{\alpha}\left(\pi_{\alpha} z\right)$ for $z \in D(S)$ and $\alpha \in$ A. Call $S$ the projective (operator) limit of $\left\{S_{\alpha}: \alpha \in A\right\} . S$ is a linear operator. If, for each $\alpha, S_{\alpha} \in L\left(Z_{\alpha}\right)$ then $S \in L_{A}(Z)$. Moreover the family $\left\{\bar{S}_{\alpha}: \alpha \in A\right\}$ associated with $S \in L_{A}(X)$ above is projective and its limit is $S$.

We conclude this section with a theorem.

\subsection{THEOREM. Let $X$ be a complete lcs. Then there is a 1-1 natural}


correspondence between $L_{A}(X)$-operators and projective families of bounded linear operators on Banach spaces.

Proof. The correspondence is nothing but the correspondence between (saturated) projective families of bounded linear operators on Banach spaces and their limits. That it is $1-1$ is a consequence of the requirement that the projective families be saturated.

2. Definition and basic properties of $\left(C_{0}, 1\right)$ semigroups.

2.1 Definition. A family $\{S(\xi): \xi \geqslant 0\} \subset L(X)$ is called a $C_{0}$ semigroup in $X$ iff

(1) $S(\xi+\eta) x=S(\xi)(S(\eta) x)$ for all $\xi, \eta \geqslant 0$ and $x \in X$;

(2) $S(0) x=x$ for all $x \in X$;

(3) $\lim _{\xi \rightarrow 0^{+}} S(\xi) x=x$ for all $x \in X$.

It is called an $L_{A}(X)$-operator semigroup of class $\left(C_{0}, 1\right)\left(\left(C_{0}, 1\right)\right.$ semigroup for short) iff, in addition, for each $\alpha$ and each positive number $\delta$, there exists a positive number $\lambda=\lambda(\alpha,\{S(\zeta): 0 \leqslant \zeta \leqslant \delta\})$ such that

$$
S(\xi) V_{\alpha} \subset \lambda V_{\alpha}, \quad 0 \leqslant \xi \leqslant \delta .
$$

2.2 Definition. A family $\{S(\xi):-\infty<\xi<\infty\} \subset L(X)$ is called a $C_{0}$ group in $X$ iff

(1) $S(\xi+\eta) x=S(\xi)(S(\eta) x)$ for all $-\infty<\xi, \eta<\infty$ and $x \in X$;

(2) $S(0) x=x$ for all $x \in X$;

(3) $\lim _{\xi \rightarrow 0^{+}} S(\xi) x=x$ for all $x \in X$.

It is a $\left(C_{0}, 1\right)$ group iff, in addition, $\{S(\xi):-\infty<\xi<\infty\} \subset L_{A}(X)$.

The next three theorems link the $\left(C_{0}, 1\right)$ (semi)group with the well-known $C_{0}$ (semi)group in Banach spaces.

2.3 Theorem. Suppose $\{S(\xi): \xi \geqslant 0\}$ is an $L_{A}(X)$-operator semigroup of class $\left(C_{0}, 1\right)$. Then, for each $\alpha$, the family $\left\{\bar{S}_{\alpha}(\xi): \xi \geqslant 0\right\}$ is a $C_{0}$ semigroup in the Banach space $\bar{X}_{\alpha}$.

Proof. Let $\alpha \in A$ be arbitrary but fixed. Now for any $x_{\alpha} \in X_{\alpha}$,

$$
S_{\alpha}(\xi+\eta) x_{\alpha}=(S(\xi+\eta) x)_{\alpha}=S_{\alpha}(\xi)(S(\eta) x)_{\alpha}=S_{\alpha}(\xi) S_{\alpha}(\eta) x_{\alpha} .
$$

Moreover for any $\bar{x}_{\alpha} \in \bar{X}_{\alpha}$, there exists a sequence $\left\{x_{\alpha}(n)\right\} \subset X_{\alpha}$ with $x_{\alpha}(n) \rightarrow \bar{x}_{\alpha}$ as $n \rightarrow \infty$. Therefore, by the continuity of $\bar{S}_{\alpha}(\zeta)(\zeta \geqslant 0)$,

$$
\begin{aligned}
\bar{S}_{\alpha}(\xi+\eta) \bar{x}_{\alpha} & =\lim _{n \rightarrow \infty} S_{\alpha}(\xi+\eta) x_{\alpha}(n)=\lim _{n \rightarrow \infty} S_{\alpha}(\xi) S_{\alpha}(\eta) x_{\alpha}(n) \\
& =\bar{S}_{\alpha}(\xi) \lim _{n \rightarrow \infty} S_{\alpha}(\eta) x_{\alpha}(n)=\bar{S}_{\alpha}(\xi) \bar{S}_{\alpha}(\eta) \lim _{n \rightarrow \infty} x_{\alpha}(n)=\bar{S}_{\alpha}(\xi) \bar{S}_{\alpha}(\eta) \bar{x}_{\alpha},
\end{aligned}
$$


which is $2.1(1)$. Similarly we have $2.1(2)$,

$$
\bar{S}_{\alpha}(0) \bar{x}_{\alpha}=\bar{x}_{\alpha} \text { for all } \bar{x}_{\alpha} \in \bar{X}_{\alpha} .
$$

Now consider 2.1(3). For each $x \in X, \lim _{\xi \rightarrow 0^{+}} S(\xi) x=x$. Hence

$$
\lim _{\xi \rightarrow 0^{+}} S(\xi) x_{\alpha}=x_{\alpha}, \quad x_{\alpha} \in X_{\alpha}
$$

Further $\left\|S_{\alpha}(\xi)\right\|=P_{\alpha}(S(\xi))$ is bounded in each interval $[0, \delta], 0<\delta<\infty$ (consequence of $1.3,1.4$ and 2.1). A $3-\epsilon$ argument then shows that (3) holds and the proof is complete.

2.4 Theorem. Suppose $\{S(\xi):-\infty<\xi<\infty\}$ is a $\left(C_{0}, 1\right)$ group. Then for each $\alpha$, the family $\left\{\bar{S}_{\alpha}(\xi):-\infty<\xi<\infty\right\}$ is a $C_{0}$ group in the Banach space $\bar{X}_{\alpha}$.

Proof. As in the foregoing proof,

(1) $\bar{S}_{\alpha}(\xi+\eta) \bar{x}_{\alpha}=\bar{S}_{\alpha}(\xi) \bar{S}_{\alpha}(\eta) \bar{x}_{\alpha},-\infty<\xi, \eta<\infty$ and $\bar{x}_{\alpha} \in \bar{X}_{\alpha}$;

(2) $\bar{S}_{\alpha}(0) \bar{x}_{\alpha}=\bar{x}_{\alpha}, \bar{x}_{\alpha} \in \bar{X}_{\alpha}$;

(3) $\lim _{\xi \rightarrow 0^{+}} S_{\alpha}(\xi) x_{\alpha}=x_{\alpha}, x_{\alpha} \in X_{\alpha}$.

Now (1)-(3) imply that for each $x_{\alpha} \in X_{\alpha}$, the vector-valued function $S_{\alpha}(\cdot) x_{\alpha}$ is strongly continuous on $(-\infty, \infty)$ and so strongly measurable there. Since $X_{\alpha}$ is dense in $\bar{X}_{\alpha}$ and $\bar{S}_{\alpha}(\xi)$ is bounded for each $\xi$, then for each $\bar{x}_{\alpha} \in \bar{X}_{\alpha}$, $\bar{S}_{\alpha}(\cdot) \bar{x}_{\alpha}$ is everywhere the strong limit of strongly measurable functions. Hence itself is strongly measurable [2, Theorem 3.5.4(3)]. Therefore $\left\|\bar{S}_{\alpha}(\xi)\right\|$ is bounded in some neighborhood of the origin [2, Lemma 10.2.1]. Again as in the foregoing proof we conclude that $\lim _{\xi \rightarrow 0}+\bar{S}_{\alpha}(\xi) \bar{x}_{\alpha}=\bar{x}_{\alpha}$ for all $\bar{x}_{\alpha} \in \bar{X}_{\alpha}$ and the proof is complete.

The group analogue of ensuing theorems will be obvious and as such will not be stated.

We call $\left\{S_{\alpha}(\xi) \in L\left(Z_{\alpha}\right): \alpha \in A, \xi \geqslant 0\right\}$ a projective family of $C_{0}$ semigroups on Banach spaces $Z_{\alpha}$ iff, for each $\xi \geqslant 0,\left\{S_{\alpha}(\xi): \alpha \in A\right\}$ is a projective family and, for each $\alpha,\left\{S_{\alpha}(\xi): \xi \geqslant 0\right\}$ is a $C_{0}$ semigroup on the Banach space $Z_{\alpha}$. Let $\{S(\xi): \xi \geqslant 0\}$ be the limit (in the obvious sense) of such a family. Let $x \in X$ and $\xi, \dot{\eta} \geqslant 0$. Then by the definition of projective limit and the linearity of $\pi_{\alpha}$,

$$
\pi_{\alpha}(S(\xi+\eta) x-S(\xi) S(\eta) x)=S_{\alpha}(\xi+\eta) x_{\alpha}-S_{\alpha}(\xi) S_{\alpha}(\eta) x_{\alpha}=0, \quad \alpha \in A .
$$

Thus $S(\xi+\eta) x=S(\xi) S(\eta) x$. Similarly $\lim _{\xi \rightarrow 0^{+}} S(\xi) x=x$. Now by the Banach space $C_{0}$ semigroup property there exists $\sigma_{\alpha}$ such that 


$$
p_{\alpha}(S(\xi) x) \stackrel{\text { def }}{=}\left\|\pi_{\alpha}(S(\xi) x)\right\|=\left\|S_{\alpha}(\xi) x_{\alpha}\right\| \leqslant e^{\sigma \alpha \xi}\left\|x_{\alpha}\right\| \leqslant e^{\sigma_{\alpha} \xi} p_{\alpha}(x) .
$$

We conclude therefore that $\{S(\xi): \xi \geqslant 0\}$ is a $\left(C_{0}, 1\right)$ semigroup.

2.5 THEOREM. There is a 1-1 correspondence between $\left(C_{0}, 1\right)$ semigroups on complete lcs and projective families of $C_{0}$ semigroups on Banach spaces such that, if $A$ is the infinitesimal generator of a $\left(C_{0}, 1\right)$ semigroup and $\left\{A_{\alpha}\right\}$ is the family of generators associated with the corresponding $C_{0}$ semigroups, then $\left\{A_{\alpha}\right\}$ is a projective family and its limit is $A$.

Proof. It is clear from above and Theorem 2.3 that a semigroup is $\left(C_{0}, 1\right)$ in a complete lcs iff it is the limit of a projective family of Banach space $C_{0}$ semigroups. Now the correspondence between a projective family $\left\{S_{\alpha}(\xi): \xi \geqslant 0\right.$, $\alpha \in A\}$ of Banach space $C_{0}$ semigroups and its limit, $\{S(\xi): \xi \geqslant 0\}$ say, is 1-1 (cf. Theorem 1.13). Therefore the first part follows. Now let $A_{\alpha}$ generate $\left\{S_{\alpha}(\xi): \xi \geqslant 0\right\}$ and $A$ generate $\{S(\xi): \xi \geqslant 0\}$. For $x_{\alpha} \in D\left(A_{\alpha}\right)$ and $\alpha \geqslant \alpha^{\prime}$,

$$
\begin{aligned}
\pi_{\alpha^{\prime} \alpha}\left(A_{\alpha} x_{\alpha}\right) & =\pi_{\alpha^{\prime} \alpha} \lim _{\xi \rightarrow 0^{+}} \frac{S_{\alpha}(\xi) x_{\alpha}-x_{\alpha}}{\xi}=\lim _{\xi \rightarrow 0^{+}} \pi_{\alpha^{\prime} \alpha}\left(\frac{S_{\alpha}(\xi) x_{\alpha}-x_{\alpha}}{\xi}\right) \\
& =\lim _{\xi \rightarrow 0^{+}} \frac{S_{\alpha^{\prime}}(\xi) x_{\alpha^{\prime}}-x_{\alpha^{\prime}}}{\xi}=A_{\alpha^{\prime} x_{\alpha^{\prime}} .}
\end{aligned}
$$

Hence $\left\{A_{\alpha}\right\}$ is a projective family. Similar arguments show that $\pi_{\alpha}(A x)=$ $A_{\alpha} x_{\alpha}$ for all $x \in D(A)$. Therefore, if the limit of $\left\{A_{\alpha}\right\}$ is $A^{\prime}$ then $A \subset A^{\prime}$. Suppose $x \in D\left(A^{\prime}\right)$. Then

$$
\begin{aligned}
p_{\alpha}\left(\frac{S(\xi) x-x}{\xi}-A^{\prime} x\right) & =\left\|\pi_{\alpha}\left(\frac{S(\xi) x-x}{\xi}-A^{\prime} x\right)\right\| \\
& =\left\|\frac{\dot{S}_{\alpha}(\xi) x_{\alpha}-x_{\alpha}}{\xi}-A_{\alpha} x_{\alpha}\right\| \rightarrow 0
\end{aligned}
$$

as $\xi \rightarrow 0^{+}$. Hence $x \in D(A)$. It follows that $A=A^{\prime}$ which proves the second and final part of the theorem.

We say that a $\left(C_{0}, 1\right)$ semigroup is of "type $\left\{\omega_{\alpha}: \alpha \in A\right\}$ " iff the $\omega_{\alpha}$ 's are the types [2, p. 306] of the associated Banach space $C_{0}$ semigroups. In particular, we say it is of "bounded type $\omega$ " if there exists a fixed real number $\omega$ such that $\sup _{\alpha} \omega_{\alpha}=\omega$.

The following theorem becomes immediate.

2.6 ThEOREM. Let a family $\{S(\xi): \xi \geqslant 0\}$ be a $C_{0}$ semigroup in $X$. Then it is an $L_{A}(X)$-operator semigroup of class $\left(C_{0}, 1\right)$ iff there exist sets $\left\{M_{\alpha}: \alpha \in A\right\}$ and $\left\{\sigma_{\alpha}: \alpha \in A\right\}$ of real numbers such that $\alpha \in A$ implies 


$$
p_{\alpha}(S(\xi) x) \leqslant M_{\alpha} e^{\sigma_{\alpha} \xi} p_{\alpha}(x) \text { for all } \xi \geqslant 0 \text { and } x \in X .
$$

Moreover, $a\left(C_{0}, 1\right)$ semigroup is strongly continuous on $[0, \infty)$.

Now consider the semigroup $\{S(\xi): \xi \geqslant 0\}$ defined as follows:

2.7 Definition. $\{S(\xi): \xi \geqslant 0\}$ is a family of continuous linear operators on $X$ into itself such that

(1) $\{S(\xi): \xi \geqslant 0\}$ is a semigroup of class $\left(C_{0}\right)$ in $X$;

(2) for each continuous seminorm $p$ on $X$ there exist a nonnegative number $\sigma_{p}$ and a continuous seminorm $q$ on $X$ such that $p(S(\xi) x) \leqslant e^{\sigma} p^{\xi} q(x)$ for all $\xi \geqslant 0$ and $x \in X$.

2.8 Theorem (CF. [7, Theorem 4]). $\{S(\xi): \xi \geqslant 0\} \subset L(X)$ is a semigroup of Definition 2.7 iff it is an $L_{A}(X)$-operator semigroup of class $\left(C_{0}, 1\right)$.

Suppose $\left\{p_{\alpha}^{\prime}: \alpha \in A\right\}$ is the family of all continuous seminorms on $X$ and $\sigma_{p_{\alpha}^{\prime}}=\sigma_{\alpha}$ is as given in Definition 2.7. Then a suitable family $\left\{p_{\alpha}: \alpha \in A\right\}$ of continuous seminorms giving rise to $L_{A}(X)$ can be constructed by defining for each $\alpha \in A$ and all $x \in X$,

$$
p_{\alpha}(x)=\sup _{\xi \geqslant 0} p_{\alpha}^{\prime}\left(e^{-\sigma_{\alpha} \xi} S(\xi) x\right) .
$$

Proof. The "if" part is clear. Now suppose $G=\{S(\xi): \xi \geqslant 0\}$ is a semigroup of Definition 2.7. Then $\left\{p_{\alpha}: \alpha \in A\right\}$ defined in the theorem gives rise to an $L_{A}(X)$ which is such that

$$
p_{\alpha}(S(\xi) x) \leqslant e^{\sigma_{\alpha} \xi} p_{\alpha}(x), \quad \alpha \in A, \xi \geqslant 0, x \in X .
$$

Thus, by Theorem 2.6, $G$ is of class $\left(C_{0}, 1\right)$.

REMARK. A $C_{0}$ semigroup on a complete lcs is equicontinuous (resp. quasi-equicontinuous) iff it is the projective limit of contraction (resp. equi-quasibounded) $C_{0}$ semigroups on Banach spaces.

3. Generators and resolvents in a $\left(C_{0}, 1\right)$ semigroup.

3.1 Definition. Let $X$ be an lcs and let $\{S(\xi): \xi \geqslant 0\}$ be a $C_{0}$ semigroup in $X$. The infinitesimal generator $A$ of $\{S(\xi): \xi \geqslant 0\}$ is defined as

$$
A x=\lim _{\xi \rightarrow 0^{+}} \frac{S(\xi) x-x}{\xi}
$$

whenever the limit exists, the domain $D(A)$ of $A$ being the set of $x \in X$ for which the limit exists.

In the case where $\{S(\xi): \xi \geqslant 0\}$ is a $\left(C_{0}, 1\right)$ semigroup, denote by $\mathscr{A}_{\alpha}$ the infinitesimal generator of the corresponding semigroup $\left\{\bar{S}_{\alpha}(\xi): \xi \geqslant 0\right\}$. 
This section deals with the infinitesimal generator $A$ of a $\left(C_{0}, 1\right)$ semigroup and its relationship with the $\hat{A}_{\alpha}$ 's.

3.2 Proposition. Let $X$ be an lcs and let $G=\{S(\xi): \xi \geqslant 0\}$ be a $\left(C_{0}, 1\right)$ semigroup with infinitesimal generator $A$ and of type $\left\{\omega_{\alpha}: \alpha \in A\right\}$ then

(1) $A$ is a closed linear operator;

(2) if $X$ is (sequentially) complete then the domain $D(A)$ of $A$ is dense in $X$;

(3) for each $\alpha, A_{\alpha}$ is a closable linear operator in $\bar{X}_{\alpha}$, the resolvent $R\left(\lambda ; \bar{A}_{\alpha}\right)\left(\lambda>\omega_{\alpha}\right)$ of its closure (i.e., its minimum closed extension) $\bar{A}_{\alpha}$ exists and there exists $M_{\alpha}$ such that

$$
\left\|\left[R\left(\lambda, \overline{A_{\alpha}}\right)\right]^{k}\right\| \leqslant M_{\alpha}\left(\lambda-\sigma_{\alpha}\right)^{-k}, \quad \lambda>\sigma_{\alpha}>\omega_{\alpha} .
$$

Proof. Consider (1) and (2). Note that (a), by definition, $G$ is a $C_{0}$ semigroup and (b), for each $x \in X, S(\cdot) x$ is continuous on $[0, \infty)$. The rest of the proof for (1) and (2) is well known. See for instance [4, pp. 260-262]. Now consider (3). Observe that $\hat{A}_{\alpha} \supset A_{\alpha}$ and $\hat{A}_{\alpha}$, being the infinitesimal generator of a $C_{0}$ semigroup in $\bar{X}_{\alpha}$, is closed. Hence $A_{\alpha}$ is closable in $\bar{X}_{\alpha}$ and $\hat{A}_{\alpha} \supset \bar{A}_{\alpha}$. It is known that $\hat{A}_{\alpha}$ satisfies (3) so if we show that $\bar{A}_{\alpha}=\hat{A}_{\alpha}$ then the proof is complete. We only need show that $D\left(\bar{A}_{\alpha}\right) \supset D\left(\hat{A}_{\alpha}\right)$ to do this. Now for, $\lambda>\omega_{\alpha}$, the resolvent $R\left(\lambda ; A_{\alpha}\right)$ of $\hat{A}_{\alpha}$ exists and is given by

$$
R\left(\lambda ; \hat{A}_{\alpha}\right) \bar{x}_{\alpha}=\int_{0}^{\infty} e^{-\lambda \xi} \bar{S}_{\alpha}(\xi) \bar{x}_{\alpha} d \xi, \quad \bar{x}_{\alpha} \in \bar{X}_{\alpha} .
$$

Thus by an argument of Hille if $x_{\alpha} \in D\left(A_{\alpha}\right)$, then $R\left(\lambda ; \hat{A}_{\alpha}\right) x_{\alpha} \in D\left(\bar{A}_{\alpha}\right)$ and $\bar{A}_{\alpha} R\left(\lambda ; \hat{A}_{\alpha}\right) x_{\alpha}=\lambda x_{\alpha}-x_{\alpha} . D\left(A_{\alpha}\right)$ is dense in $X_{\alpha}$. Hence by the closure property of $\bar{A}_{\alpha}$ and the continuity of $R\left(\lambda ; \hat{A}_{\alpha}\right), \bar{x}_{\alpha} \in \bar{X}_{\alpha}$ implies $R\left(\lambda ; \hat{A}_{\alpha}\right) \bar{x}_{\alpha} \in$ $D\left(\bar{A}_{\alpha}\right)$. Thus $R\left(\lambda ; \hat{A}_{\alpha}\right) \bar{X}_{\alpha} \subset D\left(\bar{A}_{\alpha}\right)$. But $R\left(\lambda ; \hat{A}_{\alpha}\right) \bar{X}_{\alpha}=D\left(\hat{A}_{\alpha}\right)$. Therefore the proof is complete.

It is now clear that, in a complete lcs, $\bar{A}_{\alpha}$ can replace $\hat{A}_{\alpha}$.

3.3 THEOREM. Let $X$ be a complete lcs and let $\{S(\xi): \xi \geqslant 0\}$ be a $\left(C_{0}, 1\right)$ semigroup, having $A$ as its infinitesimal generator. Suppose further that the semigroup is of bounded type $\omega$. Then the operator $R(\lambda ; A)(\lambda>\omega)$ defined by

$$
R(\lambda ; A) x=\int_{0}^{\infty} e^{-\lambda \xi} S(\xi) x d \xi \quad(x \in X),
$$

is the resolvent of $A$ for $\lambda>\omega$. Moreover (1) $R(\lambda ; A)(\lambda>\omega)$ is an $L_{A}(X)$ operator; (2) $\overline{R_{\alpha}(\lambda ; A)}=R\left(\lambda ; \bar{A}_{\alpha}\right)(\lambda>\omega, \alpha \in A)$ and (3) there exists a set $\left\{M_{\alpha}: \alpha \in \mathrm{A}\right\}$ of positive numbers such that 


$$
P_{\alpha}\left([R(\lambda ; A)]^{n}\right)=\left\|\left[R\left(\lambda ; \bar{A}_{\alpha}\right)\right]^{n}\right\| \leqslant M_{\alpha}\left(\lambda-\omega_{0}\right)^{-n}
$$

for all $\alpha \in A, \lambda>\omega_{0}>\omega$ and $n=1,2,3, \cdots$.

Proof. The proof of the first part follows similar arguments as for the equicontinuous $C_{0}$ semigroup case [13, p. 240]. We consider (1), (2), (3). As a $\left(C_{0}, 1\right)$ semigroup having bounded type $\omega$ we have each $R\left(\lambda ; \bar{A}_{\alpha}\right)(\lambda>$ $\left.\omega_{0}>\omega, \alpha \in A\right)$, is bounded with $\left\|\left[R\left(\lambda ; \bar{A}_{\alpha}\right)\right]^{n}\right\| \leqslant M_{\alpha}\left(\lambda-\omega_{0}\right)^{-n}, n=1,2$, $3, \cdots$. Now since, for $\alpha \in A, \lambda>\omega$ and $x \in X$, we have

$$
\pi_{\alpha}(R(\lambda ; A) x)=\pi_{\alpha}\left(\int_{0}^{\infty} e^{-\lambda \xi} S(\xi) x d \xi\right)=\int_{0}^{\infty} e^{-\lambda \xi} S_{\alpha}(\xi) x_{\alpha} d \xi=R\left(\lambda ; \bar{A}_{\alpha}\right) x_{\alpha},
$$

it is easy to see that (1), (2), (3) hold.

4. Generation of $\left(C_{0}, 1\right)$ semigroups. Before we establish in this section the Hille-Yosida-Phillips theorem for $\left(C_{0}, 1\right)$ semigroups, we first state a lemma needed in its proof.

4.1 Lemma. Let $A$ be a compartmentalized operator such that, for each $\alpha, A_{\alpha}$ is closable and its closure $\bar{A}_{\alpha}$ has the resolvent $R\left(\lambda ; \bar{A}_{\alpha}\right)\left(\lambda>\omega_{\alpha}\right)$. Then $\bar{\pi}_{\alpha^{\prime} \alpha}\left(R\left(\lambda ; \bar{A}_{\alpha}\right) \bar{x}_{\alpha}\right)=R\left(\lambda ; \bar{A}_{\alpha^{\prime}}\right) \bar{x}_{\alpha^{\prime}}$ whenever $\alpha \geqslant \alpha^{\prime}, \bar{x}_{\alpha} \in \bar{X}_{\alpha}$ and $\lambda>$ $\max \left(\omega_{\alpha}, \omega_{\alpha^{\prime}}\right)$.

Proof. Let $\alpha \geqslant \alpha^{\prime}, \bar{x}_{\alpha} \in \bar{X}_{\alpha}$ and $\lambda>\max \left(\omega_{\alpha}, \omega_{\alpha^{\prime}}\right)$. Then by the continuity of $\bar{\pi}_{\alpha^{\prime}}$ and the resolvent equation,

$$
\left(\lambda-\bar{A}_{\alpha^{\prime}}\right) \bar{\pi}_{\alpha^{\prime} \alpha}\left(R\left(\lambda ; \bar{A}_{\alpha}\right) \bar{x}_{\alpha}\right)=\bar{\pi}_{\alpha^{\prime} \alpha}\left(\left(\lambda-\bar{A}_{\alpha}\right) R\left(\lambda ; \bar{A}_{\alpha}\right) \bar{x}_{\alpha}\right)=\bar{\pi}_{\alpha^{\prime} \alpha}\left(\bar{x}_{\alpha}\right)=\bar{x}_{\alpha^{\prime}} .
$$

Thus $\bar{\pi}_{\alpha^{\prime} \alpha}\left(R\left(\lambda ; \bar{A}_{\alpha}\right) \bar{x}_{\alpha}\right)=\left(\lambda I-\bar{A}_{\alpha^{\prime}}\right)^{-1} \bar{x}_{\alpha^{\prime}}=R\left(\lambda ; \bar{A}_{\alpha^{\prime}}\right) \bar{x}_{\alpha^{\prime}}$ which is the lemma.

4.2 Theorem (Hille-Yosida-Phillips). Let $X$ be a complete lcs. $A$ necessary and sufficient condition for a closed linear operator $A$ to be the infinitesimal generator of a unique $\left(C_{0}, 1\right)$ semigroup is that

(1) the domain $D(A)$ of $A$ is dense in $X$;

(2) $A$ is a compartmentalized and for each $\alpha \in A$ the operator $A_{\alpha}$ is closable in $\bar{X}_{\alpha}$;

(3) for each $\alpha \in A$ there exist positive numbers $\sigma_{\alpha}, M_{\alpha}$ such that the resolvent $R\left(\lambda ; \bar{A}_{\alpha}\right)$ of the closure $\bar{A}_{\alpha}$ of $A_{\alpha}$ satisfies the condition $\left\|\left[R\left(\lambda ; \bar{A}_{\alpha}\right)\right]^{n}\right\| \leqslant M_{\alpha}\left(\lambda-\sigma_{\alpha}\right)^{-n}$ for all $\lambda>\sigma_{\alpha}$ and $n=1,2,3, \cdots$.

Proof. The necessity has been established in Theorem 3.2. We now consider the sufficiency. For each $\alpha, \bar{A}_{\alpha}$ is well defined since $A_{\alpha}$ is closable. Now $D\left(\bar{A}_{\alpha}\right)$ is dense in $\bar{X}_{\alpha}$. Therefore with condition (3) it is well known that 


$$
\exp (-\lambda \xi) \sum_{k=0}^{\infty} \frac{(\lambda \xi)^{k}\left[\lambda R\left(\lambda ; \bar{A}_{\alpha}\right)\right]^{k}}{k !} \bar{x}_{\alpha}=\bar{S}_{\alpha}^{(\lambda)}(\xi) \bar{x}_{\alpha}
$$

say, is convergent for each $\bar{x}_{\alpha} \in \bar{X}_{\alpha}, \xi \geqslant 0$ and $\lambda>\sigma_{\alpha} ;$ and that $\bar{A}_{\alpha}$ is the infinitesimal generator of a unique semigroup $\left\{\bar{S}_{\alpha}(\xi): \xi \geqslant 0\right\}$ of class $\left(C_{0}\right)$ in $\bar{X}_{\alpha}$ given by

$$
\bar{S}_{\alpha}(\xi) \bar{x}_{\alpha}=\lim _{\lambda \rightarrow \infty} \bar{S}_{\alpha}^{(\lambda)}(\xi) \bar{x}_{\alpha} \text { for all } x_{\alpha} \in \bar{X}_{\alpha}, \xi \geqslant 0 .
$$

Let $\alpha \geqslant \alpha^{\prime}$ and $\bar{x}_{\alpha} \in \bar{X}_{\alpha}$. By Lemma 4.1, $\bar{\pi}_{\alpha^{\prime} \alpha}\left(R\left(\lambda ; \bar{A}_{\alpha}\right) \bar{x}_{\alpha}\right)=R\left(\lambda ; \bar{A}_{\alpha^{\prime}}\right) \bar{x}_{\alpha^{\prime}}$ for $\lambda>\max \left(\omega_{\alpha}, \omega_{\alpha^{\prime}}\right)$. Since, in addition, $\bar{\pi}_{\alpha^{\prime} \alpha}: \bar{X}_{\alpha} \rightarrow \bar{X}_{\alpha^{\prime}}$ is continuous it follows that for each $\xi \geqslant 0$

$$
\begin{aligned}
\bar{\pi}_{\alpha^{\prime} \alpha}\left(\bar{S}_{\alpha}(\xi) x_{\alpha}\right) & =\bar{\pi}_{\alpha^{\prime} \alpha}\left(\lim _{\lambda \rightarrow \infty} \bar{S}_{\alpha}^{(\lambda)}(\xi) x_{\alpha}\right)=\lim _{\lambda \rightarrow \infty}\left(\bar{\pi}_{\alpha^{\prime} \alpha}\left(\bar{S}_{\alpha}^{(\lambda)}(\xi) x_{\alpha}\right)\right) \\
& =\lim _{\lambda \rightarrow \infty} \bar{S}_{\alpha^{\prime}}^{(\lambda)}(\xi) x_{\alpha^{\prime}}=\bar{S}_{\alpha^{\prime}}(\xi) x_{\alpha^{\prime}} .
\end{aligned}
$$

Therefore $\left\{\bar{S}_{\alpha}(\xi): \xi \geqslant 0, \alpha \in A\right\}$ is a projective family of $C_{0}$ semigroups on Banach spaces $\bar{X}_{\alpha}$ 's, and so, by Theorem 2.5 , it is associated with a unique $\left(C_{0}, 1\right)$ semigroup $\{S(\xi): \xi \geqslant 0\}$ whose infinitesimal generator is the projective operator limit of $\left\{\bar{A}_{\alpha}: \alpha \in A\right\}$ which is $A$. Now suppose $A$ generates another $\left(C_{0}, 1\right)$ semigroup $\left\{S^{\prime}(\xi): \xi \geqslant 0\right\}$. Then by Theorem 2.5 it is associated with another projective family $\left\{S_{\beta}^{\prime}(\xi)\right\}$ of $C_{0}$ semigroups whose family of generators $\left\{A_{\beta}^{\prime}\right\}$ must be distinct from $\left\{\bar{A}_{\alpha}\right\}$ since the Hille-Yosida-Phillips theorem for Banach space $C_{0}$ semigroups asserts that $C_{0}$ semigroups are uniquely generated. We conclude that the generator of $\left\{S^{\prime}(\xi): \xi \geqslant 0\right\}$ which is the projective limit of $\left\{A_{\beta}^{\prime}\right\}$ is distinct from $A$ since we are dealing with distinct saturated projective families. Hence $A$ generates a unique $\left(C_{0}, 1\right)$ semigroup and the proof is complete.

4.3 Lemma. The projective limit of closed operators is closed.

Proof. Let $A$ be the projective limit of a family $\left\{A_{\alpha}\right\}$ of closed operators. Now ( $A$ is closed) if (whenever $\exists$ a net $x(\beta) \rightarrow x$ with $A x(\beta) \rightarrow y$ then $A x=y$ ) if (whenever $\exists$ a net $x(\beta)$ with $x_{\alpha}(\beta) \rightarrow x_{\alpha}$ and $A_{\alpha} x_{\alpha}(\beta) \rightarrow$ $y_{\alpha}$ then $\left.A_{\alpha} x_{\alpha}=y_{\alpha} \forall \alpha\right)$ if ( $A_{\alpha}$ is closed for each $\alpha$ ); which proves the lemma.

4.4 THEOREM. A linear operator $A$ is the infinitesimal generator of a $\left(C_{0}, 1\right)$ semigroup on a complete lcs iff it is the projective limit of generators of $C_{0}$ semigroups in Banach spaces.

Proof. Observe that the generators of $C_{0}$ semigroups in Banach spaces 
are closed. The theorem is now an immediate consequence of Theorem 4.2 and Lemma 4.3.

4.5 Corollary. A linear operator $A$ generates an equicontinuous (resp. quasi-equicontinuous) $C_{0}$ semigroup on a complete lcs iff it is the projective limit of generators of contraction (resp. equi-quasi-bounded) $C_{0}$ semigroups on Banach spaces.

5. A perturbation theorem. In this section we treat the perturbation of the infinitesimal generator of an $L_{A}(X)$-operator semigroup of class $\left(C_{0}, 1\right)$ by an $L_{\mathrm{A}}(X)$-operator .

By the theory of perturbation for semigroups in Banach spaces developed by Phillips and the fact that the $\left(C_{0}, 1\right)$ semigroup $\{S(\xi): \xi \geqslant 0\} \equiv\{S(\xi, A): \xi \geqslant 0\}$ is such that, for each $\alpha \in A,\left\{\bar{S}_{\alpha}(\xi): \xi \geqslant 0\right\} \equiv\left\{\bar{S}_{\alpha}\left(\xi ; \bar{A}_{\alpha}\right): \xi \geqslant 0\right\}$ is a $C_{0}$ semigroup in the Banach space $\bar{X}_{\alpha}$; we have the following lemma which we shall require in the proof of the perturbation theorem.

5.1 LemmA. Let $\{S(\xi ; A): \xi \geqslant 0\}$ be an $L_{A}(X)$-operator semigroup of class $\left(C_{0}, 1\right)$ with infinitesimal generator $A$. Let $B \in L_{A}(X)$. For each $\alpha$ set

$$
\begin{aligned}
& \hat{G}_{\alpha[0]}(\xi) \bar{x}_{\alpha}=\bar{S}_{\alpha}\left(\xi ; \bar{A}_{\alpha}\right) \bar{x}_{\alpha}, \quad \xi \geqslant 0 \text { and } \bar{x}_{\alpha} \in \bar{X}_{\alpha} ; \\
& \hat{G}_{\alpha[n]}(\xi) \bar{x}_{\alpha}=\int_{0}^{\xi} \bar{S}_{\alpha}\left(\xi-\zeta ; \bar{A}_{\alpha}\right) \bar{B}_{\alpha} \hat{G}_{\alpha[n-1]}(\zeta) \bar{x}_{\alpha} d \zeta, \\
& \xi \geqslant 0 \quad(n=1,2,3, \cdots), \text { and } \bar{x}_{\alpha} \in \bar{X}_{\alpha} .
\end{aligned}
$$

Then, for each $n, \hat{G}_{\alpha[n]}(\xi)$ is strongly continuous in $\xi(\xi>0)$. Further $\left(\overline{A+B)_{\alpha}}=\bar{A}_{\alpha}+\bar{B}_{\alpha}\right.$ generates a $C_{0}$ semigroup $\left\{\bar{S}_{\alpha}\left(\xi ; \bar{A}_{\alpha}+\bar{B}_{\alpha}\right): \xi \geqslant 0\right\}$ given by

$$
\bar{S}_{\alpha}\left(\xi ; \bar{A}_{\alpha}+\bar{B}_{\alpha}\right)=\sum_{n=0}^{\infty} \hat{G}_{\alpha[n]}(\xi), \quad \xi \geqslant 0
$$

the series being uniformly absolutely convergent with respect to $\xi$ in each finite interval.

We now have the perturbation theorem.

5.2 TheOREM. Let $\{S(\xi ; A): \xi \geqslant 0\}$ be an $L_{A}(X)$-operator semigroup of class $\left(C_{0}, 1\right)$ with infinitesimal generator $A$. Suppose $B \in L_{A}(X)$. Set

$$
\begin{aligned}
& G_{[0]}(\xi)=S(\xi), \quad \xi \geqslant 0 ; \\
& G_{[n]}(\xi) x=\int_{0}^{\xi} S(\xi-\zeta ; A) B G_{[n-1]}(\zeta) x d \zeta, \\
& x \in X, \xi \geqslant 0 \text { and } n=1,2,3, \cdots .
\end{aligned}
$$


Then $A+B$ defined on $D(A)$ is also the infinitesimal generator of an $L_{\lambda}(X)$ operator semigroup $\{S(\xi ; A+B): \xi \geqslant 0\}$ of class $\left(C_{0}, 1\right)$ given by

$$
S(\xi ; A+B)=\sum_{n=0}^{\infty} G_{[n]}(\xi), \quad \xi \geqslant 0,
$$

the series being uniformly absolutely convergent with respect to $\xi$ in each finite interval.

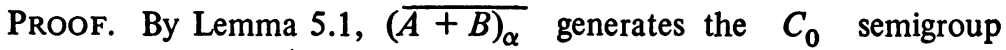
$\bar{S}_{\alpha}\left(\xi ; \bar{A}_{\alpha}+\bar{B}_{\alpha}\right)=\Sigma_{n=0}^{\infty} \hat{G}_{\alpha[n]}(\xi)$ in $\bar{X}_{\alpha}$. Since $S(\xi)$ and $B$ belong to $L_{A}(X)$ and $\bar{\pi}_{\beta \alpha}(\alpha \geqslant \beta)$ is continuous, an induction on $n$ shows that whenever, $\xi \geqslant 0$, $\bar{x}_{\alpha} \in \bar{X}_{\alpha}$ and $\alpha \geqslant \beta$,

$$
\bar{\pi}_{\beta \alpha}\left(\hat{G}_{\alpha[n]}(\xi) \bar{x}_{\alpha}\right)=\hat{G}_{\beta[n]}(\xi) \bar{x}_{\beta} .
$$

Consequently

$$
\bar{\pi}_{\beta \alpha}\left(S_{\alpha}\left(\xi ; \bar{A}_{\alpha}+\bar{B}_{\alpha}\right) \bar{x}_{\alpha}\right)=\sum_{n=0}^{\infty} \hat{G}_{\beta[n]}(\xi) \bar{x}_{\beta}=S_{\beta}\left(\xi ; \bar{A}_{\beta}+\bar{B}_{\beta}\right) \bar{x}_{\beta} .
$$

Therefore $\left\{S\left(\xi ; \bar{A}_{\alpha}+\bar{B}_{\alpha}\right): \xi \geqslant 0, \alpha \in A\right\}$ is a projective family of Banach space $C_{0}$ semigroups. The projective limit is the $\left(C_{0}, 1\right)$ semigroup, $\{S(\xi ; A+B)$ : $\xi \geqslant 0$ \} say, whose infinitesimal generator is $A+B$, the projective limit of $\left\{\overline{A+B)_{\alpha}}\right\}_{\alpha \in A}$.

Now set

$$
G(\xi)=\sum_{n=0}^{\infty} G_{[n]}(\xi), \quad \xi>0
$$

Let $\left\{\omega_{\alpha}: \alpha \in A\right\}$ be the type of $\{S(\xi): \xi \geqslant 0\}$ with $\left\{\sigma_{\alpha}: \alpha \in A\right\}$ such that $\sigma_{\alpha}>\omega_{\alpha}$.

With the aid of Lemma 5.1, the usual $L_{A}(X)$ argument and induction technique we have for $n=0,1,2, \cdots, G_{[n]}(\xi)$ is in $L_{A}(X)$ and strongly continuous on $[0, \infty)$, with

$$
P_{\alpha}\left(G_{[n]}(\xi)\right) \leqslant M_{\alpha}\left(M_{\alpha} P_{\alpha}(B)\right)^{n} \xi^{n} \exp \left(\sigma_{\alpha} \xi\right) / n ! \text { for all } \alpha \in A, \xi \geqslant 0 ;
$$

and

$$
\bar{G}_{[n] \alpha}(\xi)=\hat{G}_{\alpha[n]}(\xi) \text { for all } \alpha \in A \text { and } \xi \geqslant 0 \text {. }
$$

Thus the series (5.3) is majorized with respect to each seminorm $P_{\alpha}(\alpha \in A)$ by the series expansion of $M_{\alpha} \exp \left(\nu_{\alpha} \xi\right)$ where $\nu_{\alpha}=\sigma_{\alpha}+M_{\alpha} P_{\alpha}(B)$. The series (5.3) is therefore uniformly absolutely convergent in each finite interval. 
Moreover, for each $\alpha \in A, \xi \geqslant 0$ and $x \in X$,

$$
\begin{aligned}
\pi_{\alpha}(G(\xi) x) & =\sum_{n=0}^{\infty} \pi_{\alpha}\left(G_{[n]}(\xi) x\right)=\sum_{n=0}^{\infty} G_{[n] \alpha}(\xi) x_{\alpha} \\
& =\sum_{n=0}^{\infty} \bar{G}_{[n] \alpha}(\xi) x_{\alpha}=\sum_{n=0}^{\infty} \hat{G}_{\alpha[n]}(\xi) x_{\alpha}=\bar{S}_{\alpha}\left(\xi, \bar{A}_{\alpha}+\bar{B}_{\alpha}\right) x_{\alpha} .
\end{aligned}
$$

Therefore $G(\xi) \equiv S(\xi ; A+B)$ and the proof is complete.

6. Examples and applications. Observe that Sobolev's lemma and the usual embedding of $S$ in $C^{\infty}(\mathrm{T})$ for $\mathrm{T}$ the 1-torus shows that the $L^{2}$ estimates $p_{m n}$ (see Introduction) yield the same topology on $S$ as the usual sup-norm estimates. Now consider, in more detail, the generator $A=M D=x d / d x$ which is the vector field on $\mathbf{R}$ (associated with the group of dilations on the line and the associated operator group $G=\{S(\xi): \xi \geqslant 0\}$ defined by $(S(\xi) f)(x)=f\left(e^{\xi} x\right)$. By routine change of variables, $\|S(\xi) f\|=e^{-1 / 2 \xi}\|f\|$ and by the chain rule, $D^{n} S(\xi) f=e^{n \xi} S(\xi) D^{n} f$, while $M^{m} S(\xi) f=e^{-m \xi} S(\xi) M^{m} f$ so that $p_{m n}(S(\xi) f)=$ $e^{(n-m-1 / 2) \xi} p_{m n}(f)$. That is, $\sigma_{p_{m n}}=n-m-1 / 2$ is the best possible exponential growth rate for $p_{m n}$, so $S(\xi)$ is clearly of class $\left(C_{0}, 1\right)$ on $S$ but for no $\omega<\infty$ is $\left\{e^{-\omega|\xi|} S(\xi):-\infty<\xi<\infty\right\}$ equicontinuous.

It is clear that $G$ has no Laplace transform in $S$. Its infinitesimal generator has no resolvent either. Reason. $\lambda u-A u=0 \Rightarrow u=0$. Thus $(\lambda-A)^{-1}$ exists. However,

$$
\begin{aligned}
\lambda u-A u=f & \Rightarrow \lambda u(x)-x \frac{d}{d x} u(x)=f(x) \\
& \Rightarrow u(x)=-x^{\lambda}\left(\int s^{-(\lambda+1)} f(x) d x+\text { const }\right) .
\end{aligned}
$$

Thus

$$
D\left((\lambda-A)^{-1}\right) \subset B \stackrel{\text { def }}{=}\{f \in S: \exists 0<\delta<\infty,|x|<\delta \Rightarrow f(x)=0\} .
$$

Now take $g(x)=e^{-x^{2}}$ and any $f \in B$. Then $\left|f(x)-e^{-x^{2}}\right|_{x=0}=1$. Hence $\sup _{x \in \mathrm{R}, f \in B}\left|f(x)-e^{-x^{2}}\right| \geqslant 1$, which implies $D\left((\lambda-A)^{-1}\right)$ is not dense in $S$. Hence the lack of resolvent of $A$.

Unlike the globally or locally equicontinuous $C_{0}$ semigroup, the adjoint of a $\left(C_{0}, 1\right)$ semigroup need not be of class $\left(C_{0}, 1\right)$ even on reflexive spaces. For convenience consider the $\left(C_{0}, 1\right)$ semigroup $G=\{S(-\xi): \xi \geqslant 0\}$ which is the left half of the group $G$. $S^{\prime}$ is the dual of $S$ and $\left\langle f, f^{\prime}\right\rangle$ is the usual inner product on $S \times S^{\prime} . f_{n}^{\prime}(n=0,1,2, \cdots)$ defined by $\left\langle f, f_{n}^{\prime}\right\rangle=\int_{R} x^{2 n} f(x) d x$ $(f \in S)$ are in $S^{\prime}[11$, p. 95]. By a suitable change of variables, 


$$
\begin{aligned}
\left.K f, S^{*}(-\xi) f_{n}^{\prime}\right\rangle \mid & \left.\stackrel{\text { def }}{=} K S(-\xi) f, f_{n}^{\prime}\right\rangle|=| \int_{\mathrm{R}} x^{2 n}(S(-\xi) f)(x) d x \mid \\
& =e^{(2 n+1) \xi}\left|\int_{\mathrm{R}} x^{2 n} f(x) d x\right|=e^{(2 n+1) \xi}\left\langle f, f_{n}^{\prime}\right\rangle \mid .
\end{aligned}
$$

Set $f_{0}(x)=e^{-x^{2}}$ We assert that the weakly continuous seminorm $p(\cdot)=$ $\left\langle f_{0}, \cdot\right\rangle \mid$ on $S^{\prime}$ is such that there is no fixed number $\sigma$ and a weakly continuous seminorm $q$ on $S^{\prime}$ with $p\left(S^{*}(-\xi) f^{\prime}\right) \leqslant e^{\sigma \xi} q\left(f^{\prime}\right) \forall \xi$, $f^{\prime}$. Suppose the contrary. Then $q\left(f_{n}^{\prime}\right) \geqslant e^{(2 n+1-\sigma) \xi} p\left(f_{n}^{\prime}\right) \forall \eta$, $\xi$. But $p\left(f_{n}^{\prime}\right) \neq 0 \forall n$. Hence $q\left(f_{n}^{\prime}\right)$ is infinitely large if $n>(\sigma-1) / 2$ which is absurd. Thus the adjoint of $G_{-}$is not of class $\left(C_{0}, 1\right)$ if $S^{\prime}$ carries the weak topology or, a fortiori, the strong topology.

We now display the easy application of Theorem 4.2 by using it to show that $x d / d x$ generates a $\left(C_{0}, 1\right)$ semigroup on $S$. Observe that if $(f, g)_{m n}=$ $\left(M^{m} D^{n} f, M^{m} D^{n} g\right)$ then $A_{m n}=A-n+m+1 / 2$ satisfies $\left(A_{m n} f, g\right)_{m n}=$ $-\left(f, A_{m n} g\right)_{m n}$, whence it follows easily on the quotient Hilbert spaces that $\left\|\left(\lambda-\bar{A}_{m n}\right)^{-1}\right\| \leqslant\left|R_{e}(\lambda)\right|^{-1}$. Thus hypothesis (3) of Theorem 4.2 is satisfied. Clearly hypothesis (1) and (2) of the same theorem are satisfied. Hence the conclusion follows.

Consider a natural application of Theorem 5.2 Let $\varphi$ be a $C^{\infty}$ function on $\mathbf{R}$ possessing bounded derivatives of all orders with $(B f)(x)=\varphi(x) f(x)$. Here, let $q_{m r}=\max \left\{p_{m n}: n \leqslant r\right\}$, observing that $G$ as above is still of class $\left(C_{0}, 1\right)$. Then a combination of Leibniz rule and Schwarz inequality calculation shows that $q_{m r}(B f) \leqslant M_{r} q_{m r}(f)$ so that $A+B$ generates a $\left(C_{0}, 1\right)$ semigroup.

Consider another application. Take the operator

$$
\frac{d^{2}}{d x^{2}}+p(x) \frac{d}{d x}+q(x)
$$

on $S$ with $p$ and $q$ possessing bounded derivatives of all orders. It is not difficult to see that $d^{2} / d x^{2}$ generates a $\left(C_{0}, 1\right)$ semigroup on $S$. Now set

$$
\varphi(x)=-\frac{1}{2} \frac{d}{d x}(p(x))-\frac{(p(x))^{2}}{4}+q(x) .
$$

$\varphi$ as above possesses bounded derivatives of all orders. It follows similarly that $A=d^{2} / d x^{2}+\varphi(x)$ generates a $\left(C_{0}, 1\right)$ semigroup. Set

$$
\chi(x)=\exp \left(-\frac{1}{2} \int_{0}^{x} p(s) d s\right) .
$$

Then from elementary calculus $\chi$ and $\chi^{-1}$ are $C^{\infty}$ functions with bounded derivatives of all orders. Observe that if $A$ generates a $\left(C_{0}, 1\right)$ semigroup $P(\xi)$ on $S$ and $g \in S$ is such that $g^{-1}$ and every derivative of $g$ are bounded then ${ }^{g} A_{g}$ defined by 


$$
{ }^{g} A_{g} f=g A\left(g^{-1} f\right) \text { with } D\left({ }^{g} A_{g}\right)=\left\{f \in S: g^{-1} f \in D(A)\right\}
$$

also generates a $\left(C_{0}, 1\right)$ semigroup $T(\xi)$ given by $T(\xi) f=g P(\xi)\left(g^{-1} f\right)$ (cf. $[1$, Theorem 5.34]). We therefore conclude that

$$
\mathrm{x}_{A_{\mathrm{x}}}=\frac{d^{2}}{d x^{2}}+p(x) \frac{d}{d x}+q(x)
$$

generates a $\left(C_{0}, 1\right)$ semigroup.

\section{REFERENCES}

1. V. A. Babalola, Perturbation theory for semigroups of operators on locally convex topological spaces, Ph. D. Thesis, University of Ibadan, Nigeria, 1971.

2. E. Hille and R. S. Phillips, Functional analysis and semi-groups, rev. ed., Amer. Math. Soc. Colloq. Publ., vol. 31, Amer. Math. Soc., Providence, R. I., 1957. MR 19, 664.

3. H. Komatsu, Semi-groups of operators in locally convex spaces, J. Math. Soc. Japan 16 (1964), 230-262. MR 32 \#368.

4. T. Kômura, Semigroups of operators in locally convex spaces, J. Functional Analysis 2 (1968), 258-296. MR 38 \#2634.

5. E. A. Michael, Locally multiplicatively-convex topological algebras, Mem. Amer. Math. Soc. No. 11 (1952). MR 14, 482.

6. I. Miyadera, Semi-groups of operators in Fréchet space and applications to partial differential equations, Tôhoku Math. J. (2) 11 (1959), 162-183. MR 21 \#7445.

7. R. T. Moore, Banach algebras of operators on locally convex spaces, Bull. Amer. Math. Soc. 75 (1969), 68-73. MR 38 \#5018.

8. - Generation of equicontinuous semigroups by hermitian and sectorial operators. I, II, Bull. Amer. Math. Soc. 77 (1971), 224-229, 368-373. MR 43 \#3846a,b.

9. S. Ouchi, Semigroups of operators in locally convex spaces, J. Math. Soc. Japan 25 (1973), 265-276.

10. L. Schwartz, Lectures on mixed problems in partial differential equations and the representation of semigroups, Tata Inst. Fund. Research, Bombay, India, 1958.

11. Théorie des distributions. II, Hermann, Paris, 1959.

12. L. Waelbroeck, Les semi-groupes différentiables, Deuxième Colloq. l'Anal. Fonct., Centre Belge Recherches Math., Librairie Universitaire, Louvain, 1964, pp. 97-103.

MR 32 \#1569.

13. K. Yosida, Functional analysis, 3rd ed., Die Grundlehren der math. Wissenschaften, Band 123, Springer-Verlag, New York, 1971.

DEPARTMENT OF MATHEMATICS, UNIVERSITY OF IBADAN, IBADAN, NIGERIA

SCHOOL OF MATHEMATICS, INSTITUTE FOR ADVANCED STUDY, PRINCETON, NEW JERSEY 08540 